Pacific Journal of Mathematics

DILATION OF RAPIDLY DECREASING FUNCTIONS 


\section{DILATIONS OF RAPIDLY DECREASING FUNCTIONS}

\section{S. R. HARASYMIV}

Let $S$ be the space of rapidly decreasing indefinitely differentiable functions on the $n$-dimensional Euclidean space $R^{n}$, and suppose that $\phi \in S$. We attempt to characterize the closed vector subspace of $S$ which is generated by the set of all functions of the form

$$
\left(x_{1}, \cdots, x_{n}\right) \rightarrow \phi\left(a_{1} x_{1}+b_{1}, \cdots, a_{n} x_{n}+b_{n}\right)
$$

where $a_{1}, \cdots, a_{n}, b_{1}, \cdots, b_{n}$ are real numbers, with $a_{1}, \cdots, a_{n}$ nonzero. We also consider an analogous approximation problem in the space $S^{\prime}$ of temperate distribution on $R^{n}$.

1. Notation. We write $R^{n}$ for the $n$-dimensional Euclidean space. Addition and multiplication in $R^{n}$ are defined component-wise. If $k \leqq$ $n$ is a positive integer and $x \in R^{n}$, then we write $x_{k}$ for the $k$-th component of $x$. The set $R^{\sharp}$ is defined by $R^{\sharp}=\left\{x \in R^{n}: x_{k} \neq 0\right.$ for all $\left.k\right\}$.

The Pontryagin character group of $R^{n}$ is identified with $R^{n}$. We denote typical elements of $R^{n}$ by $x, y, \cdots$, or by $\chi, \xi, \cdots$ if we are thinking of $R^{n}$ as its own character group. If $\chi \in R^{n}$ then the bounded continuous character determined by $\chi$ is defined by

$$
x \rightarrow \exp (-2 \pi i \chi \circ x)\left(x \in R^{n}\right) \text {. }
$$

If $W$ is an open subset of $R^{n}$, then $C^{\infty}(W)$ will denote the set of functions which are defined in $W$ and are indefinitely differentiable there. $\boldsymbol{D}(W)$ will denote the set of indefinitely differentiable functions with compact support in $W . D^{\prime}(W)$ will be written for the space of distributions which have support in $W$. The space of rapidly decreasing indefinitely differentiable functions on $R^{n}$ and the space of temperate distributions on $R^{n}$ will be designated by $S$ and $\boldsymbol{S}^{\prime}$, respectively. We shall always assume that $S^{\prime}$ is equipped with the strong topology $\beta\left(\boldsymbol{S}^{\prime}, \boldsymbol{S}\right)$. For details of these spaces, see Schwartz [4] and [5].

If $f$ is a continuous function on $R^{n}$, then $Z(f)$ will denote the set of zeros of $f$.

Throughout, we use the standard notations of the calculus of $n$ variables; see, for example, Hörmander [3, p. 4]. If $k \leqq n$ is a positive integer, then $j_{k}$ will denote the projection of $R^{n}$ onto its $k$-th factor: $j_{k}(x)=x_{k}$ for all $x \in R^{n}$. In general, if $\alpha$ is a multi-index then $j^{\alpha}$ will be written for the function on $R^{n}$ which is defined by $j^{\alpha}(x)=$ $x_{1}^{\alpha_{1}} \cdots x_{n}^{\alpha_{n}}$ for all $x \in R^{n}$.

Suppose that $\phi \in \boldsymbol{D}\left(R^{n}\right)$ and that $a \in R^{\sharp}$. Then the function $\phi^{a} \in \boldsymbol{D}\left(R^{n}\right)$ which is defined by $\phi^{a}(x)=\phi(a x)\left(x \in R^{n}\right)$ is called a dilation 
of $\phi$. If $b \in R^{n}$ then the function $\phi_{b} \in D\left(R_{n}\right)$ which is defined by $\phi_{b}(x)=$ $\phi(x+b)$ is called a translate of $\phi$. The dilation $u^{a}$ and translate $u_{b}$ of an arbitrary distribution $u \in D^{\prime}\left(R^{n}\right)$ are defined as follows: we set $u^{a}(\phi)=|1 / j(a)| u\left(\phi^{a-1}\right)$ and $u_{b}(\phi)=u\left(\phi_{b}\right)$ for all $\phi \in D\left(R^{n}\right)$. It is easy to see that for any distribution $u, \operatorname{supp} u^{a}=a^{-1}$. supp $u$; and that if $u \in \boldsymbol{S}^{\prime}$ then $u^{a} \in \boldsymbol{S}^{\prime}$ and $\widehat{u}^{a}=|1 / j(a)| \widehat{u}^{a^{-1}}$. The latter statement may be verified by direct computation, or by reference to relation (5.15.14) in Edwards [1]. Finally, if $u$ is a function then $u^{a}(x)=u(a x)$ and $u_{b}(x)=u(x+b)$ for all $x \in R^{n}$.

2. Preliminaries. We gather into this section some results which we need in what follows. In our arguments we find it convenient to use the following notation. If $\psi$ is an arbitrary function in $\boldsymbol{S}$ and $w$ is an arbitrary temperate distribution then we shall write $\psi \nabla w$ for the function on $R^{\sharp}$ which is defined by $\psi \nabla w(x)=w\left(\psi^{x}\right)\left(x \in R^{\ddagger}\right)$.

Lemma 2.1. Suppose that $\eta \in \boldsymbol{S}$ and $s \in \boldsymbol{S}^{\prime}$ are such that

$$
\eta^{a} \circ s=0 \quad \text { for all } a \in R^{\sharp} .
$$

Then for each multi-index $\alpha$ it is true that

$$
D^{\alpha} \eta^{a} \circ j^{\alpha} s=0 \quad \text { for all } a \in R^{\sharp} .
$$

Proof. The proof proceeds by induction on $|\alpha|=\alpha_{1}+\cdots+\alpha_{n}$. The assertion is certainly true if $|\alpha|=0$. Thus, we assume that $\alpha>0$ and that

$$
D^{\beta} \eta^{a} \circ j^{\beta} s=0 \quad \text { for all } a \in R^{\sharp}
$$

whenever $\beta$ is a multi-index such that $|\beta|<|\alpha|$. We have to show that these assumptions entail that

$$
D^{\alpha} \eta^{a} \circ j^{\alpha} s=0 \quad \text { for all } a \in R^{\sharp} .
$$

With this end in view, let $a \in R^{\sharp}$ be arbitrary but fixed. If $\phi$ is an arbitrary function in $D\left(R^{n}\right)$ then Lemma 2.5 (b) in Harasymiv [2] tells us that the function $\eta \nabla \phi s$ is indefinitely differentiable on $R^{\sharp}$ and that

$$
\left.j^{\alpha}(a) \circ D^{\alpha}[\eta \nabla \phi s](a)=\sum_{\beta \leq \alpha} C_{\beta}^{\alpha}\left[j^{\beta} D^{\beta} \eta\right) \nabla \phi s\right](a)
$$

where $C_{\beta}^{\alpha}=\alpha ! / \beta !(\alpha-\beta) !$. If we notice that for each $\beta$ we have $\left(j^{\beta} D^{\beta} \eta\right)^{a}=j^{\beta} \circ D^{\beta} \eta^{a}$ then (2.5) may be rewritten in the form

$$
\begin{aligned}
j^{\alpha}(a) \circ D^{\alpha}[\eta \nabla \phi s](a) & =\sum_{\beta \leq \alpha} C_{\beta}^{\alpha} \circ \phi s\left(j^{\alpha} D^{\beta} \eta^{a}\right) \\
& =\sum_{\beta \leq \alpha} C_{\beta}^{\alpha} \circ D^{\beta} \eta^{a} \circ j^{\beta} s(\phi) .
\end{aligned}
$$


Now, if $\beta$ is a multi-index such that $\beta<\alpha$ then certainly $|\beta|<|\alpha|$. We may therefore invoke the inductive hypothesis (relation (2.3)) and deduce from (2.6) that

$$
D^{\alpha} \eta^{a} \circ j^{\alpha} s(\phi)=j^{\alpha}(\alpha) \circ D^{\alpha}[\eta \nabla \phi s](a) .
$$

Next we notice that, because of relation (2.1), we have for each $x \in R^{\sharp}$ the equality

$$
\begin{aligned}
\eta \nabla \phi s(x) & =\phi s\left(\eta^{x}\right) \\
& =\eta^{x} \circ s(\phi) \\
& =0 .
\end{aligned}
$$

Therefore the function $\eta \nabla \phi s$ vanishes everywhere on $R^{\sharp}$ and consequently the same must be true for each of its derivatives. If we combine this fact with relation (2.7) we find that

$$
D^{\alpha} \eta^{\alpha} \circ j^{\alpha} s(\phi)=0 \text {. }
$$

Now, the function $\phi \in \boldsymbol{D}\left(R^{n}\right)$ was arbitrarily chosen. Thus we infer from (2.8) that $D^{\alpha} \eta^{a} \circ j^{\alpha} s=0$. Since $a \in R^{\sharp}$ was arbitrarily chosen, we now conclude that (2.4) holds. This completes the proof of the validity of the inductive step and hence of the lemma.

CoRollary 2.2. Suppose that $\eta \in S$ and $s \in S^{\prime}$ are such that

$$
\eta^{a} \circ s=0 \quad \text { for all } a \in R^{\ddagger} .
$$

Then for each multi-index $\alpha$

$$
\operatorname{supp}\left(j^{\alpha} s\right) \subset \cap\left\{a \circ Z\left(D^{\beta} \eta\right): \beta \leqq \alpha, a \in R^{\sharp}\right\} .
$$

In the proof of the next lemma only, we shall use the following notation. If $s$ is a temperate distribution such that $j^{\alpha} s=0$ for at least one multi-index $\alpha$ then we shall write $q(s)$ for the nonnegative integer which is defined by $q(s)=\min \left\{|\alpha|: j^{\alpha} s=0\right\}$. Otherwise, we shall write $q(s)=\infty$.

REMARK. We notice that $\operatorname{supp} s \cap R^{\sharp} \neq \varnothing$ whenever $s$ is a temperate distribution such that $q(s)=\infty$. This is a consequence of the fact that every temperate distribution is of finite order.

Lemma 2.3. Suppose that $\eta \in \boldsymbol{S}$ and $s \in \boldsymbol{S}^{\prime}$ are such that for each multi-index $\alpha$

$$
\operatorname{supp}\left(j^{\alpha} s\right) \subset \cap\left\{a \circ Z\left(D^{\beta} \eta\right): \beta \leqq \alpha, a \in R^{\ddagger}\right\} .
$$

Then it is true that 


$$
\eta^{a} \circ s=0 \quad \text { for all } a \in R^{\sharp} \text {. }
$$

Proof. If $q(s)=\infty$ then (see the remark above) supp $s \cap R^{\sharp} \neq \varnothing$. Relation (2.9) then is easily seen to entail that $\eta=0$ on the whole of $R^{n}$; and the assertion of the lemma becomes a triviality.

It remains to show that Lemma 2.3 is true in the case when $q(s)$ is finite. We shall do this via induction on $q(s)$. More precisely, we shall use induction to demonstrate that the following statement is true for each nonnegative integer $m$.

$P_{m}$ : Let $s \in \boldsymbol{S}^{\prime}$ be a temperate distribution with $q(s)=m$. Then (2.10) is true for every function $\eta \in S$ which satisfies (2.9).

If $m=0$ there is nothing to prove. Thus, assume that $m>0$; and that $P_{k}$ is true for each nonnegative integer $k<m$. We have to show that these assumptions imply the truth of $P_{m}$.

Choose a multi-index $\alpha$ such that $|\alpha|=q(s)=m$. Since $m>0$, it follows that $\alpha_{k}>0$ for some positive integer $k \leqq n$. Without loss of generality, assume that $\alpha_{1}>0$.

Now let $\phi$ be an arbitrary function in $\boldsymbol{D}\left(R^{n}\right)$. Reference to Lemma 2.5 (b) in Harasymiv [2] shows that

$$
j_{1} D_{1}(\eta \nabla \phi s)=\eta \nabla \phi s+\left(j_{1} D_{1} \eta\right) \nabla \phi s \quad \text { on } R^{\sharp} .
$$

Next notice that $q\left(j_{1} s\right) \leqq|\alpha|-1=m-1$. In view of this, it is easy to see that the distribution $j_{1} s \in \boldsymbol{S}^{\prime}$ and the function $D_{1} \eta \in \boldsymbol{S}$ satisfy the hypotheses of $P_{k}$ for some nonnegative integer $k<m$. We may therefore appeal to the inductive hypothesis and assert that $\left(D_{1} \eta\right)^{x} \circ j_{1} s=$ 0 for all $x \in R^{\ddagger}$. This in turn entails that $\left(j_{1} D_{1} \eta\right)^{x} \circ s=0$ for all $x \in R^{\ddagger}$. Therefore for each $x \in R^{\ddagger}$

$$
\begin{aligned}
\left(j_{1} D_{1} \eta\right) \nabla \phi s(x) & =\phi s\left(\left(j_{1} D_{1} \eta\right)^{x}\right) \\
& =\left(j_{1} D_{1} \eta\right)^{x} \circ s(\phi) \\
& =0 .
\end{aligned}
$$

By virtue of this last identity, relation (2.11) may be rewritten in the form

$$
j_{1} D_{1}(\eta \nabla \phi s)=\eta \nabla \phi s \quad \text { on } R^{\sharp} \text {. }
$$

In a similar way (using in turn Lemma 2.5 (b) in Harasymiv [2] and the inductive hypothesis) it can be shown that

$$
j_{1}^{2} D_{1}^{2}(\eta \nabla \phi s)=\eta \nabla \phi s \quad \text { on } R^{\ddagger} \text {. }
$$

Relations (2.12) and (2.13) together entail that on $R^{\ddagger}$ we have the identity 


$$
\begin{aligned}
\eta \nabla \phi s & =j_{1} D_{1}(\eta \nabla \phi s) \\
& =j_{1} D_{1}\left(j_{1} D_{1}(\eta \nabla \phi s)\right) \\
& =j_{1} D_{1}(\eta \nabla \phi s)+j_{1}^{2} D_{1}^{2}(\eta \nabla \phi s) \\
& =\eta \nabla \phi s+\eta \nabla \phi s .
\end{aligned}
$$

It follows for this last identity that $\eta \nabla \phi s=0$ on $R^{\sharp}$; whence we see that for each $a \in R^{\sharp}$

$$
\begin{aligned}
\eta^{a} \circ s(\phi) & =\phi s\left(\eta^{a}\right) \\
& =\eta \nabla \phi s(a) \\
& =0 .
\end{aligned}
$$

Since $\phi \in \boldsymbol{D}\left(R^{n}\right)$ was arbitrary, we infer that (2.10) holds. This establishes the truth of $P_{m}$; and the proof of Lemma 2.3 is complete.

3. Some results about approximation. Let $\phi \in S$. We shall write $T[\dot{\phi}]$ for the closed vector subspace of $S$ generated by the set of all functions which have the form $x \rightarrow \phi(a x+b)\left(x \in R^{n}\right)$ where $a \in R^{\sharp}$ and $b \in R^{n}$. Similarly, if $u \in S^{\prime}$ then we shall write $T[u]$ for the closed vector subspace of $S^{\prime}$ generated by the set of distributions $\left\{\left(u_{b}\right)^{a}: a \in R^{\sharp}, b \in R^{n}\right\}$.

Theorem 3.1. Suppose that $\phi \in \boldsymbol{S}$ and that $\psi \in \boldsymbol{S}$. Then $\psi \in T[\phi]$ if and only if for each multi-index $\alpha$

$$
Z\left(D^{\alpha} \hat{\psi}\right) \supset \cap\left\{a \circ Z\left(D^{\beta} \hat{\phi}\right): \beta \leqq \alpha, a \in R^{\sharp}\right\} .
$$

Proof. If we bear in mind the Hahn-Banach theorem then the necessity of (3.1) is easy to verify. To establish sufficiency, consider any distribution $u \in S^{\prime}$ such that

$$
\phi^{a} * u=0 \quad \text { for all } a \in R^{\sharp} .
$$

Relation (3.2) is seen (if we remember the comment about the Fourier transform of a dilation which was made in $\S 1$ ) to entail that

$$
\hat{\phi}^{a} \circ \hat{u}=0 \quad \text { for all } a \in R^{\sharp} .
$$

Next notice that relation (3.1) implies that for each multi-index $\alpha$

$$
\cap\left\{a \circ Z\left(D^{\beta} \hat{\psi}\right): \beta \leqq \alpha, a \in R^{\sharp}\right\} \supset \cap\left\{a \circ Z\left(D^{\beta} \hat{\phi}\right): \beta \leqq \alpha, a \in R^{\sharp}\right\} .
$$

In view of (3.3) and (3.4), we may apply successively Corollary 2.2 and Lemma 2.3, and deduce that $\hat{\psi} \circ \hat{u}=0$. Hence also

$$
\psi * u=0 \text {. }
$$

An appeal to the Hahn-Banach theorem now yields the information 
that $\psi \in T[\phi]$

Theorem 3.2. Suppose that $u \in S^{\prime}$ and that $w \in S^{\prime}$. Then $w \in T[u]$ if and only if for each multi-index $\alpha$

$$
\operatorname{supp}\left(j^{\alpha} \hat{w}\right) \subset \overline{\cap\left\{a \circ \operatorname{supp}\left(j^{\alpha} \hat{u}\right): a \in R^{\sharp}\right\}} .
$$

Proof. Once again the "only if " part is obvious. It remains to establish the truth of the reversed implication; and, according to the Hahn-Banach theorem, we will have done this if we succeed in showing that

$$
w * \phi=0
$$

whenever $\phi \in \boldsymbol{S}$ is such that

$$
u^{a} * \phi=0 \quad \text { for all } a \in R^{\sharp} .
$$

Thus, suppose that $u \in S^{\prime}$ is such that (3.7) holds. Then it is clear that $\phi^{a} * u=0$ for all $a \in R^{\sharp}$. Reference to Corollary 2.2 now tells us that for each $b \in R^{\sharp}$ we have

$$
b^{-1} \circ \operatorname{supp}\left(j^{\alpha} \hat{u}\right)=\operatorname{supp}\left(j^{\alpha} \hat{u}^{b}\right) \subset \cap\left\{a \circ Z\left(D^{\beta} \hat{\phi}\right): \beta \leqq \alpha, a \in R^{\ddagger}\right\} .
$$

It now follows that for each multi-index $\alpha$

$$
\overline{\cup\left\{a \circ \operatorname{supp}\left(j^{\alpha} \hat{u}\right): a \in R^{\sharp}\right\}} \subset \cap\left\{a \circ Z\left(D^{\beta} \hat{\phi}\right): \beta \leqq \alpha, a \in R^{\sharp}\right\} .
$$

Relations (3.5) and (3.8) allow us to invoke Lemma 2.3 and assert that $\hat{\phi} \circ \hat{w}=0$; whence we conclude that (3.6) holds.

4. An alternative form of the results. Theorems 3.1 and may be phrased in such a way that Fourier transform do not figure explicitly in their statement. We give below this alternative form of our previous results.

THEOREM 4.1. Suppose that $\phi \in S$ and that $\psi \in S$. Then $\psi \in T[\phi]$ if and only if the following statement is true:

If $\left\{n_{1}, \cdots, n_{k}\right\}$ is a subset of $\{1,2, \cdots, n\}$ and $\left\{\alpha_{1}, \cdots, \alpha_{k}\right\}$ is a set nonnegative integers such that

$$
\int_{R} \cdots \int_{R} x_{n_{1}}^{\beta_{1}} \cdots x_{n_{k}}^{\beta_{k}} \phi\left(x_{1}, \cdots, x_{n}\right) d x_{n_{1}} \cdots d x_{n_{k}}=0
$$

everywhere for each set $\left\{\beta_{1}, \cdots, \beta_{k}\right\}$ of nonnegative integers such that $\beta_{1} \leqq \alpha_{1}, \cdots, \beta_{k} \leqq \alpha_{k}$, then

$$
\int_{R} \cdots \int_{R} x_{n_{1}}^{\alpha_{1}} \cdots x_{n_{k}}^{\alpha_{k}} \psi\left(x_{1}, \cdots, x_{n}\right) d x_{n_{1}} \cdots d x_{n_{k}}=0
$$


everywhere.

Proof. We first prove the "if" part. To this end, we assume that (4.2) is always implied by (4.1). We have to show that this assumption entails that condition (3.1) in the statement of Theorem 3.1 is satisfied.

Thus, let $\alpha$ be a multi-index; and let $\chi \in \cap\left\{a \circ Z\left(D^{\beta} \hat{\phi}\right): \beta \leqq \alpha, a \in R^{\sharp}\right\}$. Without any loss of generality, assume that $\chi_{1}=\cdots=\chi_{k}=0$ and $\chi_{k+1} \neq 0, \cdots, \chi_{n} \neq 0$. It is easy to verify that this entails that $\xi \in \cap\left\{Z\left(D^{\beta} \hat{\phi}\right): \beta \leqq \alpha\right\}$ whenever $\xi \in R^{n}$ is such that $\xi_{1}=\cdots=\xi_{k}=0$. It follows that if $\left\{\beta_{1}, \cdots, \beta_{k}\right\}$ is a set of nonnegative integers such that $\beta_{1} \leqq \alpha_{1}, \cdots, \beta_{k} \leqq \alpha_{k}$, and $\left\{\xi_{k+1}, \cdots, \xi_{n}\right\}$ is an arbitrary set of real numbers, then

$$
\begin{aligned}
\int_{R} \cdots & \int_{R} x_{1}^{\beta_{1}} \cdots x_{k}^{\beta_{k}} \phi\left(x_{1}, \cdots, x_{n}\right) \\
& \times \exp \left[-2 \pi i\left(\xi_{k+1} x_{k+1}+\cdots\right)\right] d x_{1} \cdots d x_{n} \\
= & (-i)^{\beta_{1}+\cdots+\beta_{k}} D_{1}^{\beta_{1}} \cdots D_{k}^{\beta_{k}} \hat{\phi}\left(0, \cdots, 0, \xi_{k+1}, \cdots, \xi_{n}\right) \\
= & 0 .
\end{aligned}
$$

In view of (4.3), we infer that (4.1) holds for each set $\left\{\beta_{1}, \cdots, \beta_{k}\right\}$ of nonnegative integers such that $\beta_{1} \leqq \alpha_{1}, \cdots, \beta_{k} \leqq \alpha_{k}$. Our initial assumption now allows us to assert that (4.2) holds; whence it follows readily that $\chi \in Z\left(D^{\alpha} \hat{\psi}\right)$. Since $\chi \in \cap\left\{a \circ Z\left(D^{\beta} \hat{\phi}\right): \beta \leqq \alpha, a \in R^{\sharp}\right\}$ was arbitrary, we conclude that condition (3.1) in the statement of Theorem 3.1 is indeed satisfied.

The "only if" portion of Theorem 4.1 may be established by carrying out an analogous computational argument and then using Theorem 3.1; or, alternatively, we may employ the Hahn-Banach theorem directly and reach the same conclusion.

Theorem 4.2. Suppose that $u \in \boldsymbol{S}^{\prime}$ and that $w \in \boldsymbol{S}^{\prime}$. Then $w \in T[u]$ if and only if $D^{\alpha} w=0$ whenever $\alpha$ is a multi-index such that $D^{\alpha} u=0$.

Proof. Suppose that $D^{\alpha} w=0$ whenever $\alpha$ is a multi-index such that $D^{\alpha} u=0$; but suppose that, contrary to Theorem 3.2 , there exists a multiindex $\beta$ such that $\operatorname{supp}\left(j^{\beta} \hat{w}\right) \not \subset \overline{U\left\{a \circ \operatorname{supp}\left(j^{\beta} \hat{u}\right): a \in R^{\ddagger}\right\}}$. Then we may choose $\chi \in \operatorname{supp}\left(j^{\beta} \hat{w}\right)$ such that

$$
\chi \notin \overline{U\left\{a \circ \operatorname{supp}\left(j^{\beta} \widehat{u}\right): a \in R^{\ddagger}\right\}} .
$$

Without loss of generality, assume that $\chi_{1} \neq 0, \cdots, \chi_{k} \neq 0$ and $\chi_{k+1}=$ $\cdots=\chi_{n}=0$.

We assert that 


$$
\operatorname{supp}\left(j^{\beta} \widehat{u}\right) \subset Z\left(j_{1}\right) \cup \cdots \cup Z\left(j_{k}\right) .
$$

For if there were a $\xi \in \operatorname{supp}\left(j^{3} \hat{u}\right)$ such that $\xi \in \bigcap_{r=1}^{k}\left[R^{n} \backslash Z\left(j_{r}\right)\right]$ then we would have $\xi_{1} \neq 0, \cdots, \xi_{k} \neq 0$; and it would follow that

$$
\left.\chi \in \overline{\cup\left\{a \xi: a \in R^{\sharp}\right.}\right\} \subset \overline{U\left\{a \circ \operatorname{supp}\left(j^{\beta} \hat{u}\right): a \in R^{\sharp}\right\}}
$$

which would contradict (4.4).

Now recall that every temperate distribution is of finite order. In view of this, relation (4.5) ensures the possibility of choosing a set $\left\{\alpha_{1}, \cdots, \alpha_{k}\right\}$ of nonnegative integers such that $j_{1}^{\alpha_{1}} \cdots j_{k}^{\alpha_{k} \circ j^{3}} \hat{u}=0$. It then follows that

$$
D_{1}^{\alpha_{1}} \cdots D_{k}^{\alpha_{k}} \circ D^{3} u=0 .
$$

On the other hand, $j_{1}^{\alpha_{1}} \cdots j_{k}^{\alpha_{k}}(\chi)=\chi_{1}^{\alpha_{1}} \cdots \chi_{k}^{\alpha_{k}} \neq 0$. Since $\chi \in \operatorname{supp}\left(j^{3} \hat{w}\right)$, we conclude that $\chi \in \operatorname{supp}\left(j_{1}^{\alpha_{1}} \cdots j_{k}^{\alpha_{k}} \circ j^{3} \widehat{w}\right)$. Thus

$$
D_{1}^{\alpha_{1}} \cdots D_{k_{k}}^{\alpha_{k}} \circ D^{\beta} w \neq 0 \text {. }
$$

Relations (4.6) and (4.7) together contradict our initial assumption. Hence we infer that condition (3.5) in the statement of Theorem 3.2 is satisfied, and that $w \in T[u]$.

The "only if" part of Theorem 4.2 may be obtained as an immediate consequence of either Theorem 3.2 or the Hahn-Banach theorem.

\section{BIBLIOGRAPHY}

1. R. E. Edwards, Functional analysis: theory and applications, Holt, Rinehart and Winston, New York, 1965.

2. S. R. Harasymiv, A note on approximation of distributions by quasi-analytic functions, (to appear, J. Austral. Math. Soc.)

3. L. Hörmander, Linear partial differential operators, Springer-Verlag, Heidelberg, 1963.

4. L. Schwartz, Théorie des distributions, Tome I, Hermann, Paris, 1950.

5. - Théorie des distributions, Tome II, Hermann, Paris, 1951.

Received February 16, 1968.

UNIVERSITY OF CONNECTICUT 


\section{PACIFIC JOURNAL OF MATHEMATICS}

\section{EDITORS}

\author{
H. ROYDEN \\ Stanford University \\ Stanford, California \\ Richard Pierce \\ University of Washington \\ Seattle, Washington 98105
}

\author{
J. DugundJI \\ Department of Mathematics \\ University of Southern California \\ Los Angeles, California 90007 \\ BASIL GoRDON \\ University of California \\ Los Angeles, California 90024
}

\section{ASSOCIATE EDITORS}
E. F. BECKENBACH
B. H. Neumann
F. WOLF
K. YoSHIDA

\section{SUPPORTING INSTITUTIONS}

\author{
UNIVERSITY OF BRITISH COLUMBIA \\ CALIFORNIA INSTITUTE OF TECHNOLOGY \\ UNIVERSITY OF CALIFORNIA \\ MONTANA STATE UNIVERSITY \\ UNIVERSITY OF NEVADA \\ NEW MEXICO STATE UNIVERSITY \\ OREGON STATE UNIVERSITY \\ UNIVERSITY OF OREGON \\ OSAKA UNIVERSITY \\ UNIVERSITY OF SOUTHERN CALIFORNIA
}

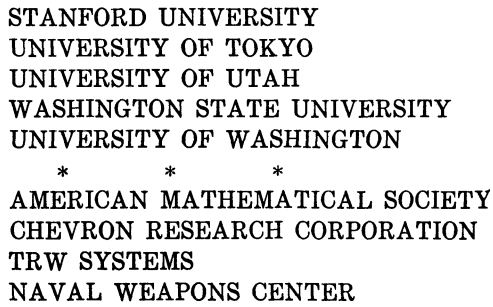

The Supporting Institutions listed above contribute to the cost of publication of this Journal, but they are not owners or publishers and have no responsibility for its content or policies.

Mathematical papers intended for publication in the Pacific Journal of Mathematics should be in typed form or offset-reproduced, double spaced with large margins. Underline Greek letters in red, German in green, and script in blue. The first paragraph or two must be capable of being used separately as a synopsis of the entire paper. It should not contain references to the bibliography. Manuscripts, in duplicate if possible, may be sent to any one of the four editors. Please classify according to the scheme of Math. Rev. 36, 1539-1546. All other communications to the editors should be addressed to the managing editor, Richard Arens, University of California, Los Angeles, California, 90024.

50 reprints are provided free for each article; additional copies may be obtained at cost in multiples of 50 .

The Pacific Journal of Mathematics is published monthly. Effective with Volume 16 the price per volume (3 numbers) is $\$ 8.00$; single issues, $\$ 3.00$. Special price for current issues to individual faculty members of supporting institutions and to individual members of the American Mathematical Society: $\$ 4.00$ per volume; single issues $\$ 1.50$. Back numbers are available.

Subscriptions, orders for back numbers, and changes of address should be sent to Pacific Journal of Mathematics, 103 Highland Boulevard, Berkeley, California, 94708.

PUBLISHED BY PACIFIC JOURNAL OF MATHEMATICS, A NON-PROFIT CORPORATION

Printed at Kokusai Bunken Insatsusha (International Academic Printing Co., Ltd.), 7-17, Fujimi 2-chome, Chiyoda-ku, Tokyo, Japan. 


\section{Pacific Journal of Mathematics}

\section{Vol. 31, No. $2 \quad$ December, 1969}

Efraim Pacillas Armendariz, Quasi-injective modules and stable torsion

classes..........................................

J. Adrian (John) Bondy, On Ulam's conjecture for separable graphs...

Vasily Cateforis and Francis Louis Sandomierski, On commutative rings over which the singular submodule is a direct summand for every module .....

Rafael Van Severen Chacon, Approximation of transformations with continuous

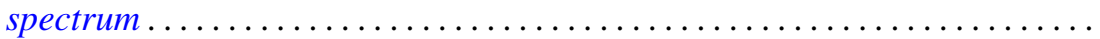

Raymond Frank Dickman and Alan Zame, Functionally compact spaces ...... 303

Ronald George Douglas and Walter Rudin, Approximation by inner

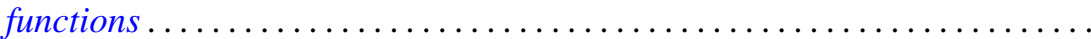

John Walter Duke, A note on the similarity of matrix and its conjugate

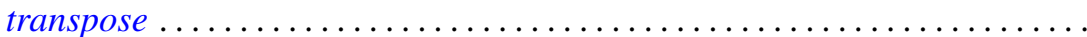

Micheal Neal Dyer and Allan John Sieradski, Coverings of mapping

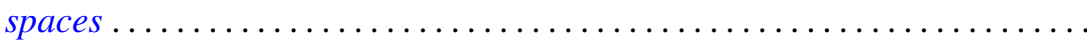

Donald Campbell Dykes, Weakly hypercentral subgroups of finite groups .....

Nancy Dykes, Mappings and realcompact spaces.....................

Edmund H. Feller and Richard Laham Gantos, Completely injective

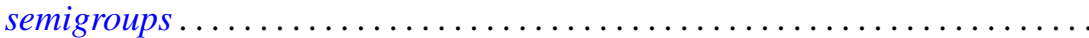

Irving Leonard Glicksberg, Semi-square-summable Fourier-Stieltjes

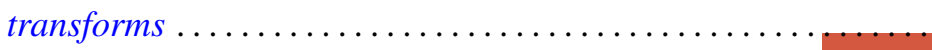

Samuel Irving Goldberg and Kentaro Yano, Integrability of almost cosymplectic structures...

Seymour Haber and Charles Freeman Osgood, On the sum $\sum\langle n \alpha\rangle^{-t}$ and numerical integration ..........................

Sav Roman Harasymiv, Dilations of rapidly decreasing functions ....

William Leonard Harkness and R. Shantaram, Convergence of a sequence of

transformations of distribution functions

Herbert Frederick Kreimer, Jr., A note on the outer Galois theory of rings ...

James Donald Kuelbs, Abstract Wiener spaces and applications to analysis. .

Roland Edwin Larson, Minimal $T_{0}$-spaces and minimal $T_{D}$-spaces...

A. Meir and Ambikeshwar Sharma, On Ilyeff's conjecture .

Isaac Namioka and Robert Ralph Phelps, Tensor products of compact convex sets....

James L. Rovnyak, On the theory of unbounded Toeplitz operators ....

Benjamin L. Schwartz, Infinite self-interchange graphs.......

George Szeto, On the Brauer splitting theorem...

Takayuki Tamura, Semigroups satisfying identity $x y=f(x$,

Kenneth Tolo, Factorizable semigroups .................. 